Review Article

\title{
The Diagnosis and Treatment of Penile Cancer
}

\author{
Oliver Walther Hakenberg, Desiree Louise Dräger, \\ Andreas Erbersdobler, Carsten Maik Naumann, Klaus-Peter Jünemann, Chris Protzel
}

\begin{abstract}
Summary
Background: The incidence of penile cancer in Europe lies in the range of 0.9 to 2.1 cases per 100000 persons per year. Carcinogenesis is associated with human papilloma virus (HPV) infection and with chronic inflammation.
\end{abstract}

Methods: This review is based on publications (2010-2017) retrieved by a selective search in PubMed and EMBASE and on the guidelines of the European Association of Urology, the European Society of Medical Oncology, the National Comprehensive Cancer Network, and the National Institute for Health and Care Excellence (NICE).

Results: $95 \%$ of cases of penile cancer are accounted for by squamous cell carcinoma, whose numerous subtypes have different clinical courses. Chronic preputial inflammation due to phimosis or lichen sclerosus is often associated with penile cancer. Circumcision lowers the risk of penile cancer (hazard ratio: 0.33 ). Maximally organ-preserving surgery with safety margins of no more than a few millimeters is the current therapeutic standard, because a local recurrence, if it arises, can still be treated locally with curative intent. Local radiotherapy can be performed in early stages. Lymphogenic metastasis must be treated with radical lymphadenectomy and adjuvant chemotherapy. Patients with clinically unremarkable inguinal lymph nodes nonetheless need invasive lymph node staging because of the high rate of lymphogenic micrometastasis.

Conclusion: Penile cancer is curable in all early stages with the appropriate treatment, but its prognosis depends crucially on the proper management of the regional (i.e., inguinal) lymph nodes. In many countries, the treatment of this rare disease entity has been centralized.

\section{Cite this as:}

Hakenberg OW, Dräger DL, Erbersdobler A, Naumann CM, Jünemann KP, Protzel C: The diagnosis and treatment of penile cancer. Dtsch Arztebl Int 2018; 115: 646-52. DOI: 10.3238/arztebl.2018.0646

Department of Urology, University Medical Center Rostock; Rostock, Germany: Prof. Dr. med. Oliver Walther Hakenberg, Dr. med. Desiree Louise Dräger,

Prof. Dr. med. Chris Protzel Institute of Pathology, University Medical Center Rostock, Ro-

stock, Germany: Prof. Dr. med. Andreas Erbersdobler

Department of Urology and Pediatric Urology, SchleswigHolstein University Hospital (UK-SH), Campus Kiel, Kiel, Germany: Prof. Dr. med. Carsten Maik Naumann,

Prof. Dr. med.

Klaus-Peter

Jünemann
D enile cancer is an aggressive squamous cell carcinoma of the skin of the glans or of the inner layer of the prepuce, characterized by invasive growth and early metastatic spread to lymph nodes. While penile cancer is uncommon in Europe, incidence rates are very high in parts of South America and Africa. Since its treatment is often associated with significant cosmetic and functional defects, the disease is of critical importance to the affected men. Early metastatic spread to regional lymph nodes can be life-threatening. It is not uncommon that factors, both from the patient and the treating physician, are causing delays in diagnosis and start of treatment

With penile cancer being a comparatively rare disease, many physicians are unfamiliar with its management. Thus, several countries have centralized the treatment of this rare tumor. Penile cancer is an orphan disease. Due the low numbers of patients, no prospective randomized studies have become available. Most of the available data is from small retrospective studies; larger studies result from retrospective multicenter data collections. Thus, the level of evidence reached for penile cancer based on the literature is consistently low. For the same reasons, pharmaceutical companies which sponsor studies are not interested in penile cancer.

This article summarizes the current knowledge of the disease and the management strategies for penile cancer. The aim of this paper is to highlight the complexity of this cancer and to show that delayed or incorrect treatment can be life-threatening. For this end, the current versions of all available guidelines on penile cancer (European Association of Urology [EAU] (9), European Society of Medical Oncology [ESMO], National Comprehensive Cancer Network [NCCN], National Institute for Health and Care Excellence [NICE], and existing Cochrane reviews as well as the respective cited literature were included in our review. In addition, an updated search of the literature was performed in the databases PubMed, EMBASE and Cochrane Database of Systematic Reviews for the period from 2010 to 2017, using the search terms "penile or penis, penile neoplasms, penile cancer". 


\section{Pathology and pathogenesis of penile cancer}

Of all penile malignancies, 95\% are squamous cell carcinomas; about half of these originate from nonkeratinized epithelium of the glans or the inner layer of the prepuce. Other malignancies (melanoma, sarcoma) or metastases are extremely rare at the penis.

In penile squamous cell carcinoma, various histologic subtypes are distinguished based on the classification of the Union Internationale Contre le Cancer (UICC) (Table 1) (1). These subtypes differ in terms of their histologic and molecular genetic characteristics, pathogenesis and prognosis.

There are two different pathogenic pathways involved in the development of penile carcinomas. About one third of cases is associated with human papilloma virus (HPV) infection. Immunohistochemical detection of p16 is used as a surrogate parameter for HPV association (2). The most commonly identified HPV serotypes are HPV 16, 18, 31, 33, 45, 56, and 65. HPV-associated penile cancer can be distinguished from non-HPV-associated types by means of polymerase chain reaction (PCR) or immunohistochemistry.

The second pathogenic pathway is chronic inflammation, associated with, for example, lichen sclerosus or chronic inflammation of the foreskin related to phimosis. Because of these two different pathogenic pathways, the new UICC classification distinguishes between HPV-associated and non-HPV-associated penile carcinomas (1).

Thus, epidemiologically confirmed risk factors include phimosis and chronic inflammation (balanoposthitis; hazard ratio [HR] 9.5; phimosis HR 3.5). Circumcision in childhood significantly reduces the prevalence of the condition (HR 0.33) (3). However, the removal of the foreskin also reduces the exposed surface of the non-keratinized penile skin by $50 \%$. In epidemiological studies, chronic cigarette smoking is described as another risk factor (HR 2.8) (4-6).

Molecular biological changes may be relevant to prognosis. In penile cancer, loss of heterozygosity $(\mathrm{LOH})$ adjacent to tumor suppressor genes $(2 \mathrm{q}, 6 \mathrm{p}$, $8 \mathrm{q}, 9 \mathrm{p}, 12 \mathrm{q}, 17 \mathrm{p} 13$ ) is commonly observed and occurs even more frequently in lymph nodes metastases $(3 p$, $6 \mathrm{p}, 6 \mathrm{q}, 8 \mathrm{q}, 9 \mathrm{p}, 11 \mathrm{q}, 12 \mathrm{q}, 15 \mathrm{q}, 17 \mathrm{p}, 18 \mathrm{q})$ (3). Particularly common are losses of alleles in the regions $9 \mathrm{p} 21$ und $17 \mathrm{p}$, coding the tumor suppressor genes p16 and p53, respectively (3). Inactivation of p16 and p53 is also effected by the HPV oncogenes E6 and E7. P16 promoter hypermethylation and $\mathrm{LOH}$ as well as other changes of other tumor suppressor genes (KAI1, $\mathrm{nm} 23 \mathrm{H} 1$ ) are associated with metastatic spread. However, whether p53 alterations are of prognostic relevance has not yet been conclusively established $(4,5)$.

The most prevalent histologic type, accounting for about 70 to $75 \%$ of cases, is the "common" squamous cell carcinoma with or without keratinization (7). It is an aggressive tumor characterized by early metastatic spread. The second most common type, accounting

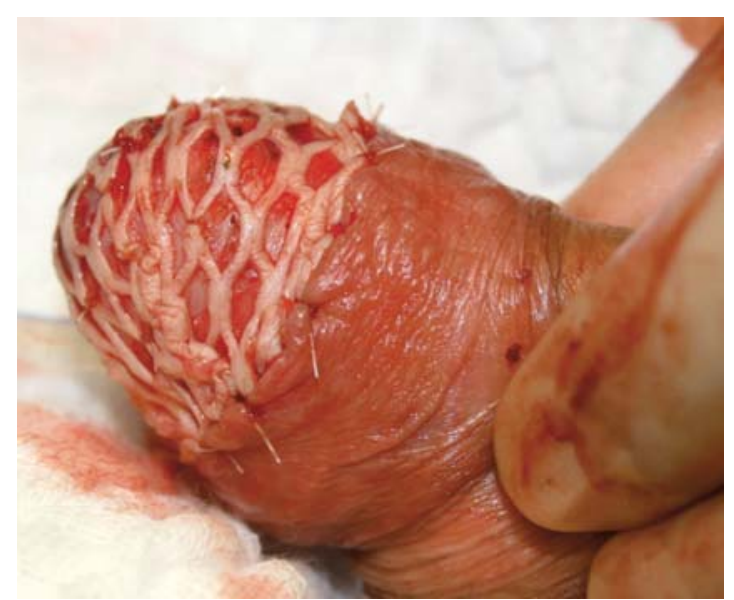

Figure 1: Glans resection with split-thickness skin graft closure

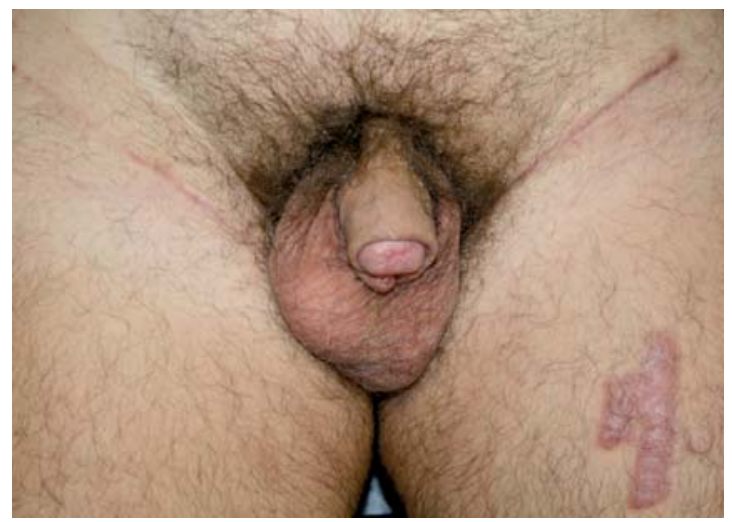

Figure 2: One year after glans resection for PT2G3 penile cancer with split-thickness skin graft repair and bilateral inguinal lymphadenectomy (pN1 bilaterally) and adjuvant chemotherapy

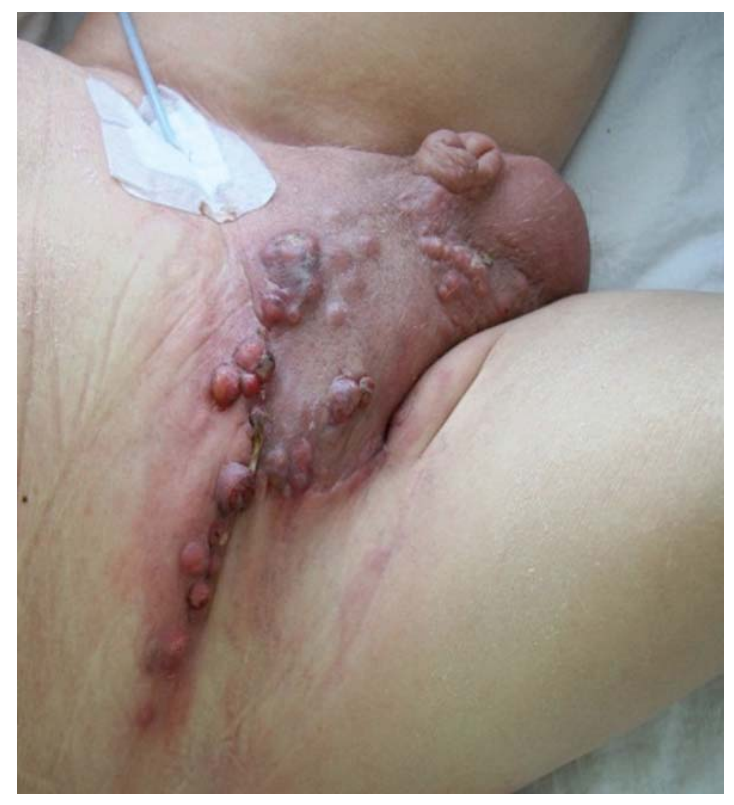

Figure 3: Extensive fixed inguinal lymph node metastases in penile cancer (N3) 
TABLE 1

WHO classification of penile carcinomas, their relative frequency and mean cancer-specific mortality $(1,2,32)$

\begin{tabular}{l|c|c}
\hline Squamous cell carcinomas & $\begin{array}{c}\text { Relative } \\
\text { frequency }\end{array}$ & $\begin{array}{c}\text { Tumor-specific } \\
\text { mortality }\end{array}$ \\
\hline Non-HPV-associated & \multicolumn{2}{|c}{} \\
\hline $\begin{array}{l}\text { Squamous cell carcinoma, } \\
\text { common type }\end{array}$ & $70-75 \%$ & $30 \%$ \\
\hline Pseudohyperplastic carcinoma & & $0 \%$ \\
\hline Pseudoglandular carcinoma & $2-3 \%$ & $>50 \%$ \\
\hline Verrucous carcinoma & & low \\
\hline Carcinoma cuniculatum & $5-8 \%$ & low \\
\hline Papillary carcinoma, NOS & rare & low \\
\hline Adenosquamous carcinoma & $1-4 \%$ & $75 \%$ \\
\hline Sarcomatoid carcinoma & & \\
\hline HPV-associated & $5-10 \%$ & $>50 \%$ \\
\hline Basaloid carcinoma & rare & \\
\hline Papillary basaloid carcinoma & $5-10 \%$ & low \\
\hline Warty carcinoma & & $30 \%$ \\
\hline Warty basaloid carcinoma & & $20 \%$ \\
\hline Clear-cell carcinoma & & not known \\
\hline Lymphoepithelioma-like carcinoma & & \\
\hline
\end{tabular}

for approximately $10 \%$ of cases, is the basaloid subtype which is very aggressive, as are the sarcomatoid and warty subtypes. By contrast, the verrucous and condylomatous subtypes only spread in exceptional cases and have a much better prognosis (Table 1) (2).

\section{Epidemiology}

In Europe and North America, the incidence of penile cancer is approximately 1.0 new case per 100000 population (7). In Sweden, the incidence is higher with comprehensive data collection $(2,1)$. In Germany, altogether 940 new cases were recorded in 2014, with a mortality of 195 cases and a mean age of onset of 70 years (3). However, penile cancer also occurs in significantly younger men, starting age 30 years.

In some developing countries, penile cancer represents a serious public health problem. The incidence rates in Central and South America, parts of Asia and Africa are significantly higher (Brazil 6-8/100 000). In rural regions in India, penile cancer accounts for up to $6 \%$ of all male cancer cases; in Uganda, the cumulative morbidity among men up to age 75 years is $1 \%(5)$. These peculiarities have been attributed to shortcomings in the healthcare system and lack of hygiene, high rates of sexually transmitted infections and a high rate of phimosis. Patient-related delays in diagnosis and treatment are not rare and associated with low socioeconomic status and low level of education (5).
Population-based analyses from Europe and the United States have shown that, in contrast to other types of cancer, the tumor-specific survival rates for penile cancer have not shown any improvement since 1990 (6).

\section{Diagnosis}

Patients note changes of the glans or foreskin, but experience no pain. In many cases, the diagnosis of exophytic penile cancer is established by inspection. Superficial stages of penile cancer (pTis, pTa [Table 2]) are often limited to surface changes. Early suspicion and biopsy are necessary to prevent delays in treatment initiation.

\section{Pathological processing, grading and staging}

Confirmation of the diagnosis by biopsy and tumor staging are both required for treatment planning. Invasive penile cancer typically shows exophytic growth. Histologic subtype and tumor grade are key determinants of prognosis. The UICC classification categorizes the grades I to III and the sarcomatoid, dedifferentiated type (1).

Grading is more difficult with squamous cell carcinomas compared to adenocarcinomas. This explains the high interobserver variability in the grading of penile cancer (8). Consequently, there is no definitive prognostic difference between G1 and G2 in penile cancer, especially since highly differentiated penile squamous cell carcinomas can show invasive growth and metastatic spread, too.

Pathological processing of the specimen should be undertaken with great care. It is important to distinguish between stage $\mathrm{T} 1 \mathrm{a}$ and stage $\mathrm{T} 1 \mathrm{~b}$. This requires special expertise for discriminating between the two can be challenging due to lymphovascular invasion or poor degree of differentiation (G3). Lymph node evaluation is of great prognostic significance because extracapsular spread of lymph node metastases - which is classified as $\mathrm{pN} 3$ even with only 1 lymph node-ultimately requires adjuvant chemotherapy (Table 2) (2).

\section{Management}

For the management of penile cancer, interdisciplinary guidelines of varying quality are available (EAU [9], ESMO, NCCN, NICE $[10,11])$. Several European countries (United Kingdom, Sweden, Denmark, the Netherlands) have centralized the management of penile cancer. With this approach, the interval between diagnosis and treatment was significantly shortened in Denmark, and in Sweden it led to improvements in guideline adherence $(12,13)$. Having established national penile cancer registries, these countries are in the position to collect data which can be used to improve treatment strategies.

\section{Stage-adapted treatment}

Especially for early stages limited to the foreskin or glans, treatment alternatives, with equal effectiveness, 
TNM classification of penile carcinomas of the Union Internationale Contre le Cancer (UICC) (32)

\begin{tabular}{|l|l|}
\hline Primary tumor $(\mathrm{T})$ & Carcinoma in situ \\
\hline Ta & Non-invasive localized verrucous carcinoma \\
\hline T1 & Tumor invades subepithelial connective tissue \\
\hline T1a & Without lymphovascular invasion and well differentiated \\
\hline T1b & With lymphovascular invasion or poorly differentiated \\
\hline T2 & Tumor invades corpus spongiosum with or without invasion of the urethra \\
\hline T3 & Tumor invades corpus cavernosum without or with invasion of the urethra \\
\hline T4 & Tumor invades other adjacent structures \\
\hline
\end{tabular}

\begin{tabular}{|l|l|l|}
$\begin{array}{l}\text { Regional lymph nodes (N) } \\
\text { (clinical) }\end{array}$ & N0 & No palpable or visibly enlarged inguinal lymph nodes \\
\hline N1 & Palpable mobile unilateral lymph node \\
\hline N2 & Palpable mobile multiple or bilateral inguinal lymph nodes \\
\hline N3 & Fixed inguinal nodal mass or pelvic lymphadenopathy, unilateral or bilateral \\
\hline
\end{tabular}

\begin{tabular}{|c|c|c|}
\hline \multirow{4}{*}{$\begin{array}{l}\text { Regional lymph nodes (pN) } \\
\text { (pathological) }\end{array}$} & pNO & No regional lymph node metastasis \\
\hline & $\mathrm{pN} 1$ & Metastasis in up to two regional lymph nodes \\
\hline & $\mathrm{pN} 2$ & Metastases in three or more unilateral lymph nodes or bilateral inguinal lymph nodes \\
\hline & $\mathrm{pN} 3$ & $\begin{array}{l}\text { Metastasis in pelvic lymph nodes, unilateral or bilateral, or extranodal extension of } \\
\text { any regional lymph node metastasis }\end{array}$ \\
\hline \multirow[t]{2}{*}{ Distant metastasis (M) } & Mo & No distant metastasis \\
\hline & M1 & Distant metastasis \\
\hline
\end{tabular}

are available. The patient should receive in-depth advice and detailed information. However, ultimately treatment decisions should be based on the wishes of the patient.

Surgical treatment of penile cancer is guided by the following principle: as much organ preservation as possible and as much radicality as necessary. Penile cancer limited to the inner layer of the prepuce is treated by "radical circumcision". In case of superficial tumors of the glans which are limited to the epithelium (pTis, pTa), the glans should be spared. This can be achieved using focal chemotherapy or immunotherapy, laser ablation, radiation therapy or surgery. Carcinoma in situ (pTis) can be treated successfully in half of the cases using topical immunotherapy or chemotherapy (imiquimod, 5fluorouracil, applied as an aqueous solution) (14). Alternative treatment options - also for recurrence or persistence of the lesion - to be considered include laser (CO2, Neodym:YAG) ablation, complete removal of the epithelium of the glans ("glans resurfacing") or radiation therapy. Here, it is important to keep in mind that superficial tumors often show invasive growth only at isolated points of the lesion
(15). The success of focal treatment should be confirmed by a follow-up biopsy. In most cases, local recurrences occur within 1 to 2 years after the initial treatment, most frequently after laser ablation (10-48\%), less frequently after glans resurfacing $(0-6 \%)$ and very rarely after glansectomy (0-2\%) (16-18).

Current recommendations for surgical treatment allow very narrow tumor-negative margins as long as a complete excision of the tumor is achieved (19). Today, the wide negative margins of up to $2 \mathrm{~cm}$ at the penis shaft recommended in the past are considered completely obsolete. Since a local recurrence as such is not a threat to the life of the patient, as it is curable by renewed local treatment (20), the most recent strategy is to try to achieve narrow, but clearly tumornegative margins to ensure optimum quality of life is maintained. Guidelines recommend to decide the required width of the tumor-negative margin based on the grading ( $1 \mathrm{~mm}$ for G1, $5 \mathrm{~mm}$ for G3) (9). Diverse surgical techniques are available to treat the various stages of invasive penile cancer. Invasive tumors of the glans (pT1, pT2) are treated by local excision, partial resection of the glans or amputation of the glans. 
While it is possible to achieve satisfactory cosmetic results by glans repair using a split-thickness skin graft or a buccal mucosa graft (Figure 1), the repaired glans will have no sensory innervation.

Larger tumors with invasion of the corpus spongiosum (pT2) or the corpora cavernosa (pT3) require glans amputation, in some cases including removal of the tips of the corpora. In these cases, plastic reconstruction should also be attempted.

Extensive tumors (pT4) require extensive amputation (partial amputation of the penis) or radical penectomy with complete removal of the corpora cavernosa (up to the insertion on the pelvic bones). In these cases, reconstructive techniques are challenging and should only be attempted in the interval if curative treatment is possible.

\section{Role of radiation therapy}

Squamous cell carcinomas are generally radiosensitive tumors. Thus, penile carcinomas can in principle be treated by percutaneous radiotherapy or focal brachytherapy. However, due to the lack of data on the radiosensitivity of the various types of penile squamous cell carcinomas, no differentiated treatment with radiation therapy can be administered.

While radiation therapy offers the potential advantage of sparing the morphological integrity of the organ, radiation-related functional impairments of the corpora cavernosa and, with meatus stenosis being a common complication (10-35\%), of urination are unavoidable (21). Local radiation therapy is a recommended option for tumors up to a maximum size of $4 \mathrm{~cm}$ in the stages T1 and T2 (4). Local brachytherapy achieves lower local control rates compared to surgical treatment (70-90\% versus $90-92 \%$ and $94-96 \%$ for glansectomy and glans resurfacing, respectively) $(14,15,22-25)$. With the majority of cases of penile cancer being treated surgically, data on radiation therapy are scarce.

\section{Regional lymph nodes}

Due to the tendency for early lymphatic metastasis, treatment of regional (inguinal and pelvic) lymph nodes is critical for prognosis. Approximately $20 \%$ of patients have palpable inguinal lymph nodes at the time of diagnosis (26). Diagnostic assessment of regional lymph nodes is usually limited to clinical examination (finding of palpable inguinal lymph nodes). However, in obese patients clinical differentiation can be challenging; in this situation, ultrasound evaluation of inguinal lymph nodes can be advantageous (20).

\section{Non-enlarged (non-palpable) inguinal lymph nodes}

The management of patients with unremarkable inguinal lymph nodes on physical examination is particularly challenging because in up to 20 to $25 \%$ of casesdepending on local stage and degree of differentiation of the tumor-inguinal lymphatic micrometastases $(0.2$ to $2 \mathrm{~mm}$ in diameter) are present (27). If left untreated, a regional lymph node recurrence will occur within a period of 1 to 2 years which has a detrimental effect on prognosis (long-term survival $<40 \%$ ).

In patients with clinically unremarkable inguinal lymph node status, diagnostic imaging does not improve the detection of lymph node metastases measuring less than $1 \mathrm{~cm}$ in diameter. Consequently, in patients with clinically unremarkable inguinal lymph nodes, invasive diagnostic investigations are performed starting from stage pT1 and grade G2-3 The two methods used for this end are:

- dynamic sentinel lymph node biopsy (DSNB) using technetium-labeled nanocolloid and patent blue dye (PBD) or

- (diagnostic) modified inguinal lymph node dissection.

For DSNB, high micrometastasis detection rates (sensitivity approximately 90-95\%) along with rates of false-negative results of 5 to $10 \%$ as well as low morbidity have been described (28).

\section{Enlarged inguinal lymph nodes}

In patients with inguinal lymph nodes suspicious on palpation, surgical removal, histologic confirmation by means of intraoperative frozen-section analysis, and, in case of positive findings, radical inguinal lymphadenectomy are indicated. Radical inguinal lymph node dissection is mandatory since it is not sufficient to only remove lymph nodes with macroscopic involvement. Prolonged attempts of antibiotic treatment for presumed inflammatory enlargement of inguinal lymph nodes are contraindicated (5). If involvement of two or more lymph nodes is detected on one inguinal side, it is necessary to perform additional ipsilateral pelvic lymphadenectomy (9). While penile cancer is characterized by bilateral lymphatic spread to the groins, metastasis from inguinal to pelvic lymph nodes is strictly ipsilateral; thus, unilateral radical pelvic lymph node dissection is sufficient in these cases (27).

Inguinal lymph node dissection is associated with considerable morbidity in the form of lymphedema, lymphoceles and complications of wound healing. In older series, this morbidity reached levels of up to $50 \%$, but advances in surgical technique have led to a reduction to a rate of approximately $25 \%$ (Figure 2) $(17,18)$. Minimally invasive surgical techniques for inguinal lymph node dissection (laparoscopic, robotassisted) are associated with lower morbidity (19).

\section{Adjuvant therapy for lymph node metastasis}

After radical lymphadenectomy, adjuvant chemotherapy improves tumor-specific survival (29-31). Depending on the extent of lymph node metastasis and the patient's comorbidities, 4 to 6 cycles of adjuvant chemotherapy are required to achieve this survival benefit.

Adjuvant inguinal lymph node irradiation is not recommended by any of the guidelines due to the lack of relevant data (9-11). Only for adjuvant irradiation of the pelvis after surgical removal of pelvic lymph 


\section{Key messages}

- Penile squamous cell carcinoma includes numerous histologic subtypes, some of which are HPV-associated, and is frequently an aggressive tumor.

- Surgical treatment is based upon the principle: as much radicality as necessary, as much organ preservation as possible.

- The treatment of regional inguinal lymph nodes is decisive for the prognosis.

- Non-enlarged inguinal lymph nodes contain micrometastases in up to $25 \%$ of cases and require, depending on their stage, invasive surgical evaluation.

- In cases with limited lymph node metastasis, radical lymphadenectomy with adjuvant chemotherapy represents a potentially curative treatment option.

node metastases, a minor survival benefit was reported.

\section{Management of advanced stages}

Locally advanced tumor stages can be treated with palliative intention either by radical surgery or radiation therapy. In patients with large inguinal nodal masses (cN3), operability can be achieved if they respond to neoadjuvant chemotherapy, ultimately resulting in long-term survival rates of up to 50\% (Figure 3) (32).

In patients with systemic metastasis (most commonly to the lungs, liver and brain), palliative chemotherapy can achieve a limited survival benefit. Second-line therapies are available, but have not been sufficiently evaluated yet. In principle, several chemotherapeutic agents are effective in penile cancer. First, the comparatively toxic Dexeus regimen was established, combining cisplatin, methotrexate and bleomycin (33). Improved tolerability was then offered by the Pizzocaro regimen (vinblastine, methotrexate, bleomycin) (34). Today, taxanecontaining regimens have been established. In $\mathrm{Eu}-$ rope, the combination of paclitaxel, cisplatin and 5-fluoruracil is preferred (35), in the United States the TIP regimen (paclitaxel, ifosfamide, cisplatin) (36).

New systemic therapies have shown little success in the treatment of penile cancer. Sorafenib and sunitinib are without effect (23); for some PD-1 inhibitors, data are available, showing a limited response (24).

\section{Outcomes and prognosis}

The overall 5-year relative survival rates were $97 \%$ for pTis/pTa tumors, $90 \%$ for pT $1,66 \%$ for pT $2,55 \%$ for pT3, and $46 \%$ for patients with positive lymph node status (pN1-3) (13). If limited lymphatic metastasis is properly treated, the prognosis remains good (37). In most cases, local recurrences respond well to treatment and have little negative impact on prognosis for survival (20). In case of a lymph node recurrence, tumor- specific survival deteriorates to levels below $40 \%$ (20). Extensive lymph node metastasis can only be cured if the patients responds well to neoadjuvant chemotherapy (38). The prognosis for patients with systemic metastasis remains extremely poor.

\section{HPV carcinogenesis and vaccination}

By analogy with the vaccination in girls, it is reasonable to assume that HPV vaccination in boys can prevent the development of a portion of penile cancer (as well as anal cancer), even though no related data have yet become available.

\section{Conflict of interest statement}

The authors declare that no conflict of interest exists.

Manuscript received on 3 December 2017; revised version accepted on 4 June 2018

Translated from the original German by Ralf Thoene, MD.

\section{References}

1. Brierley JD, Gospodarowicz MK, Wittekind C: TNM classification of malignant tumours. Union for International Cancer Control; Oxford: John Wiley \& Sons 2017.

2. Erbersdobler A: [Pathology and histopathological evaluation of penile cancer]. Urologe 2018; 57: 391-7.

3. Robert Koch-Institut und Gesellschaft der epidemiologischen Krebsregister in Deutschland e. V: Krebs in Deutschland für 2013/2014. Berlin 2017. www.krebsdaten.de/Krebs/DE/Content/ Publikationen/Krebs in Deutschland/kid 2017/krebs in deutschland_2017.pdf?_blob=publicationFile (last accessed on 8 August 2018)

4. de Martel C, Plummer M, Vignat J, et al.: Worldwide burden of cancer attributable to HPV by site, country and HPV type. Int J Cancer 2017, 141: $664-70$

5. Attalla K, Paulucci DJ, Blum K, et al.: Demographic and socioeconomic predictors of treatment delays, pathologic stage, and survival among patients with penile cancer: a report from the national cancer database. Urol Oncol 2018; 36: 14 e17-14, e24.

6. Verhoeven RH, Janssen-Heijnen ML, Saum KU, et al.: Populationbased survival of penile cancer patients in Europe and the United States of America: no improvement since 1990. Eur J Cancer 2013; 49: 1414-21.

7. Compérat E: Pathology of penile cancer. Eur Urol Suppl 2018; 17 : 132-7.

8. Kakies C, Lopez-Beltran A, Comperat E, et al.: Reproducibility of histopathologic tumor grading in penile cancer-results of a European project. Virchows Arch 2014; 464: 453-61.

9. Hakenberg OW, Comperat EM, Minhas $S$, et al.: EAU guidelines on penile cancer: 2014 update. Eur Urol 2015; 67: 142-50.

10. van Poppel H, Watkin NA, Osanto S, et al.: Penile cancer: ESMO clinical practice guidelines for diagnosis, treatment and follow-up. Ann Oncol 2013; (Suppl 6): 115-24.

11. Clark PE, Spiess PE, Agarwal $N$, et al.: Penile cancer: clinical practice guidelines in oncology. J Natl Compr Canc Netw 2013; 11: 594-615.

12. Jakobsen JK, Jensen JB: DaPeCa-2: implementation of fast-track clinical pathways for penile cancer shortens waiting time and accelerates the diagnostic process-a comparative before-and-after study in a tertiary referral centre in Denmark. Scand J Urol 2016; 50: 80-7.

13. Kirrander P, Sherif A, Friedrich B, et al.: Swedish National Penile Cancer Register: incidence, tumour characteristics, management and survival. BJU Int 2016; 117: 287-92.

14. Manjunath $A$, Brenton $T$, Wylie $S$, et al.: Topical therapy for non-invasive penile cancer (Tis)-updated results and toxicity. Transl Androl Urol 2017; 6: 803-8

15. Shabbir M, Muneer A, Kalsi J, et al.: Glans resurfacing for the treatment of carcinoma in situ of the penis: surgical technique and outcomes. Eur Urol 2011; 59: 142-7.

16. Imamura M, MacLennan S, Lam TBL, et al.: Surgical management for localised penile cancer. Cochrane Database Syst Rev 2015; 3 : CD011533.

17. Chipollini J, Yan S, Ottenhof SR, et al.: Surgical management of penile carcinoma in situ: results from an international collaborative study and review of the literature. BJU Int 2018; 121: 393-8. 
18. Tang $\mathrm{DH}$, Yan S, Ottenhof SR, et al.: Glansectomy as primary management of penile squamous cell carcinoma: an international study collaboration. Urology 2017; 109: 1040-3.

19. Minhas $S$, Kayes $O$, Hegarty $P$, et al.: What surgical resection margins are required to achieve oncological control in men with primary penile cancer? BJU Int 2005; 96: 1040-3.

20. Leijte JA, Kirrander P, Antonini N, et al.: Recurrence patterns of squamous cell carcinoma of the penis: recommendations for follow-up based on a two-centre analysis of 700 patients. Eur Urol 2008; 54 $161-8$

21. Zouhair A, Coucke PA, Jeanneret W, et al.: Radiation therapy alone or combined surgery and radiation therapy in squamous-cell carcinoma of the penis? Eur J Cancer 2001; 37: 198-203.

22. Crook J, Ma C, Grimard L: Radiation therapy in the management of the primary penile tumor: an update. World J Urol 2009; 27: 189-96.

23. Li J, Zhu Y, Zhang SL, et al.: Organ-sparing surgery for penile cancer: complications and outcomes. Urology 2011; 78: 1121-4.

24. Crook J, Grimard L, Pond G, et al.: Penile brachytherapy: results for 60 patients. Urology 2007; 70: 161-5.

25. Escande A, Haie-Meder C, Mazeron R, et al.: Brachytherapy for conservative treatment of invasive penile carcinoma: prognostic factors and long-term analysis of outcome. Int J Radiat Oncol Biol Phys 2017; 99: 563-70.

26. Persson B, Sjödin JG, Holmberg L, et al.: The national penile cancer register in Sweden 2000-2003. Scand J Urol Nephrol 2007; 41: 278-82.

27. O'Brien JS, Perera M, Manning T, et al.: Penile cancer: contemporary lymph node management. J Urol 2017; 197: 1387-95.

28. Zou ZJ, Liu ZH, Tang LY, et al.: Radiocolloid-based dynamic sentinel lymph node biopsy in penile cancer with clinically negative inguinal lymph node: an updated systematic review and meta-analysis. Int Uro Nephrol 2016; 48: 2001-13

29. Necchi A, Pond GR, Raggi D, et al.: Clinical outcomes of perioperative chemotherapy in patients with locally advanced penile squamous-cell carcinoma: results of a multicenter analysis. Clin Genitourin Cancer 2017; 15: 548-55.

30. Necchi A, Lo Vullo S, Nicolai N, et al.: Prognostic factors of adjuvant taxane, cisplatin, and 5-fluorouracil chemotherapy for patients with penile squamous cell carcinoma after regional lymphadenectomy. Clin Genitourin Cancer 2016; 14: 518-23.

31. Nicolai N, Sangalli LM, Necchi A, et al.: A combination of cisplatin and 5 -fluorouracil with a taxane in patients who underwent lymph node dissection for nodal metastases from squamous cell carcinoma of the penis: treatment outcome and survival analyses in neoadjuvant and adjuvant settings. Clin Genitourin Cancer 2016; 14: 323-30.

32. Dickstein RJ, Munsell MF, Pagliaro LC, et al.: Prognostic factors influencing survival from regionally advanced squamous cell carcinoma of the penis after preoperative chemotherapy. BJU Int 2016; 117: 118-25.

33. Hakenberg OW, Nippgen JB, Froehner M, et al.: Cisplatin methotrexate and bleomycin for treating advanced penile carcinoma BJU Int 2006; 98: 1225-7.

34. Pizzocaro G, Nicolai N, Milani A: Taxanes in combination with cisplatin and fluorouracil for advanced penile cancer: preliminary results. Eur Urol 2009; 55: 546-51.

35. Pizzocaro G, Piva L: Adjuvant and neoadjuvant vincristine, bleomycin, and methotrexate for inguinal metastases from squamous cell carcinoma of the penis. Acta Oncol 1988; 27: 823-4.

36. Pagliaro LC, Williams DL, Daliani D, et al.: Neoadjuvant paclitaxel ifosfamide, and cisplatin chemotherapy for metastatic penile cancer: a phase II study. J Clin Oncol 2010; 28: 3851-7.

37. Djajadiningrat RS, Graafland NM, van Werkhoven E, et al: Contemporary management of regional nodes in penile cancer-improvement of survival? J Urol 2014; 191: 68-73.

38. Necchi A, Lo Vullo S, Perrone F, et al.: First-line therapy with dacomitinib, an orally available pan-HER tyrosine kinase inhibitor, for locally advanced or metastatic penile squamous cell carcinoma: results of an open-label, single-arm, single-centre, phase 2 study. BJU Int 2018; 121: 348-56.

\section{Corresponding author}

Prof. Dr. med. Oliver Walther Hakenberg

Universitätsmedizin Rostock, Urologie

Schillingallee 35

18057 Rostock, Germany

oliver.hakenberg@med.uni-rostock.de

\section{-O CLINICAL SNAPSHOT}

\section{A 27-Year-Old Man With Abruptly Occurring Chest Pain on Swallowing}

A 27-year-old man complained of sudden, left-sided, respiration-related thoracic pain every time he swallowed food or drink. He was known to have a history of chronic renal insufficiency with $\lg A$ nephropathy and well-controlled bronchial asthma. His vital signs and electrocardiographic findings were normal, but a rale was detected on palpation of the neck. Thoracic radiography and subsequent computed tomography show marked pneumomediastinum with pneumopericardium, a small, left-sided ventral pneumothorax, and right-sided cervical soft-tissue emphysema. No contrast medium was observed in the mediastinum. Esophagogastroscopy showed intact mucosae. The patient received symptomatic treatment, and radiological follow-up 5 days later revealed clear regression of the pneumomediastinum with residual cervical air pockets. Spontaneous pneumomediastinum is a relatively rarely occur-

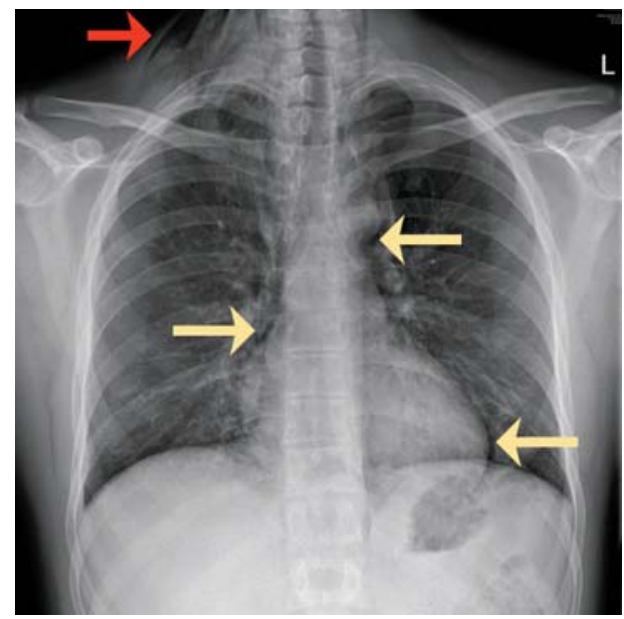
The thoracic radiograph shows pneumomediastinum with pneumopericardium (yellow arrows) and marked rightsided cervical softtissue emphysema (red arrow). ring entity (estimated incidence 1:7000 to 1:45 000) that is seen most frequently in young men and is generally self-limiting. Endoscopy is not absolutely necessary in a clinically stable patient.

PD Dr. Radovan Vasko, Prof. Gerhard Anton Müller, Klinik für Nephrologie und Rheumatologie, Georg-August-Universität Göttingen, vaskorad@gmail.com

Conflict of interest statement: The authors declare that no conflict of interest exists.

Translated from the original German by David Roseveare

Cite this as: Vasko R, Müller GA: A 27-year-old man with abruptly occurring chest pain on swallowing. Dtsch Arztebl Int 2018; 115: 652. DOI: 10.3238/arztebl.2018.0652 